\title{
Neuroimaging tests for clinical psychiatry: Are we there yet?
}

\author{
Marco Leyton, PhD; Sidney H. Kennedy, MD
}

Biomarkers index normal and abnormal biological processes, sometimes identifying the response potential of particular treatments. Though widely used in much of medicine, none has proven sufficiently robust to enter clinical practice in psychiatry. ${ }^{1,2}$ And yet, recent high-quality neuroimaging studies give confidence that this is not an unattainable goal. Here's why.

\section{Neural fingerprinting}

There is now replicated evidence of neural "fingerprints." These functional connectivity networks are unique to the individual and consistent across testing conditions., In the largest of these studies, with nearly 800 participants tested between the ages of 8 and 22 years, networks stabilized earlier in female than in male participants and earlier in healthy adolescents than psychologically troubled ones. ${ }^{4}$ Individual differences in these "fingerprints" show evidence of being shaped by early life experiences ${ }^{5,6}$ and of corresponding to cognitive-affective traits. ${ }^{6,7}$

\section{Mood}

There is now tantalizing evidence that brain connectivity patterns could help predict treatment responses in patients with mood disorders. ${ }^{8-12}$ In a large multisite study ${ }^{10}$ with a sample size of more than 1000 patients, 2 configurations were identified. One was characterized by psychomotor retardation and anhedonia linked to altered frontostriatal connectivity and diverse associations with the orbitofrontal cortex. The second was characterized by symptoms of insomnia and anxiety linked to limbic region connectivity (amygdala, hippocampus, ventral striatum, subgenual cingulate, lateral prefrontal cortex). Together these orthogonal clusters yielded 4 "biotypes" that remained stable upon rescanning 4-6 weeks later. These biotypes also predicted treatment responses in a patient subsample $(n=124)$ that was administered repetitive transcranial magnetic stimulation (rTMS). Though placebo controls were absent, treatment responses ranged from $25 \%$ to $83 \%$, with the combination of symptoms plus biotype classifying response with greater than $90 \%$ accuracy. When additional functional MRI (fMRI) quality controls were implemented the level of accuracy increased further. Connectomebased strategies have also been applied to other treatment modalities, and distinct subgenual cingulate resting state functional connectivity patterns have been identified in patients ( $n=122$ ) who responded to cognitive behavioural therapy versus an antidepressant. ${ }^{11}$ In each case, the effects were large enough to suggest clinical utility with the promise of benefits to patients and health care services.

\section{Addictions}

Altered connectivity patterns ${ }^{13}$ and regionally specific activations ${ }^{14}$ have both been tentatively identified in association with addictions. In a meta-analysis of studies measuring responses to monetary reward, differences were observed in people with substance use disorders as compared to healthy volunteers. ${ }^{14}$ During the reward anticipation phase, arguably a form of secondary conditioning, people with addictions exhibited elevated activations in the amygdala and diminished

\section{Definitions}

Biotype: A biologically distinct phenotype usually assumed to be closely related to a genotype.

Cingulo-opercular network: Includes the dorsal anterior cingulate and anterior insula as connectivity hubs, and influences attentional processing and goal-directed behaviours.

Endophenotype: A state-independent heritable trait. The evidence for psychiatric endophenotypes is weak, but personality traits remain candidates. See also the study by Clementz and colleagues ${ }^{32}$ (2016).

Global efficiency: An index of global network efficiency (high efficiency = few mediating nodes). It has been associated with IQ and general cognitive function.

Local efficiency: An index of local network efficiency. Like global efficiency, it covaries with cognitive function.

Secondary conditioning: A cue predicts a cue that predicts an unconditioned stimulus.

Subcortical network: The primary network hubs are in the thalamus and basal ganglia.

Correspondence to: M. Leyton, Department of Psychiatry, McGill University, 1033 Pine Ave West, Montreal, QC H3A 1A1; marco.leyton@mcgill.ca 
responses in the striatum. During the reward outcome phase (e.g., "You win money!"), considered a complex mix of conditioned and unconditioned reward, people with addictions exhibited augmented responses in the limbic striatum.

It might also be possible to identify markers of future addictions. As found for mood disorders, there is evidence of separable subtypes. In 759 emerging adults (average age $19.6 \pm 1.2$ years), 2 distinct markers of stress-related problem drinking were identified. ${ }^{15}$ One group exhibited elevated threat-induced activations of the amygdala coupled with reduced reward-related activations of the ventral striatum. The second group exhibited the converse pattern: elevated reward-related activations in the ventral striatum and diminished threat-induced activations in the amygdala. The former effects were mediated by anxious-depressive symptoms, while the latter were mediated by delay-discounting impulsivity. We have identified strikingly similar effects. Anxietysensitive individuals exhibited elevated amygdalar responses to threat images, and these responses were reduced by alcohol ingestion. In comparison, high sensation-seeking participants exhibited increased stress-induced activations of the medial orbitofrontal cortex and ventral striatum, with the former effect diminished by alcohol ingestion. In both subgroups, prospective follow-up data showed that individual differences in the magnitude of response, specific to each risk group, predicted who transitioned to a substance use problem (M.L., unpublished data, 2017).

The origins of these pathway-specific neurobiological alterations remain unclear, but altered mesocorticolimbic reactivity might include dysregulated dopamine ${ }^{16}$ and glutamate transmission. ${ }^{17}$ The heightened amygdalar responses in anxious individuals might be potentiated by histories of adverse life experiences ${ }^{18}$ and reflect compromised habituation processes when threatening stimuli are presented repeatedly without negative consequences. ${ }^{19}$

\section{Psychoses}

Connectivity disturbances have long been hypothesized in patients with schizophrenia, though the particulars were elusive. Now, however, there is replicated evidence that patients with psychotic features exhibit a consistent pattern of reduced global efficiency within the cingulo-opercular salience and subcortical networks $(n=576){ }^{20}$ Individual differences in network efficiency covaried with cognitive function, with the cortical and the subcortical networks making statistically independent contributions. These findings replicate observations made in 2 separate smaller samples ${ }^{21,22}$ and were presaged by similar findings from independent researchers. ${ }^{23}$

\section{What next?}

The excitement generated by these observations should not obscure the challenges ahead. Normative ranges will need to be defined and replicated across different scanners and sites. Care will be required to ensure that the information obtained does not promote prejudicial profiling. And fMRI data are susceptible to movement and other artifacts, raising the possibility that, even if the identified markers prove reliable, they may be unrelated to etiology. ${ }^{24}$

These cautions noted, there is reason for optimism. First, procedures for reducing artifacts are improving rapidly, and the studies described here are of generally high methodological quality (e.g., see Appendix 2, Figure S1 of the study by Drysdale and colleagues ${ }^{10}$ ). Second, work in other areas of medicine suggests that biomarkers with even greater precision might be constructed from multidimensional indices, including combinations of genes, molecules and systems. ${ }^{25}$ For diseases of the brain too, the ability to predict better (or worse) outcomes has been achieved by collating multiple markers, with higher numbers of markers performing better. ${ }^{26-29}$ This strategy might be effective in psychiatry also. ${ }^{30}$ Endophenotypes constructed from multiple social, clinical and brain volumetric alterations identified subgroups of patients that crossed diagnoses $(n=1872) .{ }^{31}$ The combination of symptoms plus connectivity patterns predicted responses to rTMS better than either feature alone..$^{10}$

As anticipated, the tentatively identified biomarkers do not map on to conventional diagnostic categories. ${ }^{1,32}$ For example, Drysdale and colleagues ${ }^{10}$ replicated the anxietyinsomnia-related depression biotype in patients with diagnoses of generalized anxiety disorder. The studies by Sheffield and colleagues ${ }^{20-22}$ found the same psychosis-related connectivity patterns, irrespective of whether the patient's formal diagnosis was schizophrenia, schizoaffective disorder, or a bipolar mood disorder. Mary Phillips' research group reports that, in patients with bipolar depression, emotional dysregulation is associated with disturbed connectivity in the limbic circuitry implicated in negative mood states; ${ }^{9}$ high sensation-seeking youth exhibit frontostriatal connectivity alterations across diagnoses. ${ }^{33}$ Together, this rapidly accumulating body of work suggests that it is no longer incautious to propose that psychiatric biomarkers are within our reach, taking us closer to precision diagnoses, prognoses and treatment.

Affiliations: From the Department of Psychiatry and Psychology, McGill University, Montreal, Que., Canada (Leyton); the Department of Neurology \& Neurosurgery, Montreal Neurological Institute, McGill University, Montreal, Que., Canada (Leyton); the Center for Studies in Behavioral Neurobiology, Concordia University, Montreal, Que., Canada (Leyton); the Research Unit on Children's Psychosocial Maladjustment, Université de Montréal, Montreal, Que., Canada (Leyton); the Canadian Biomarker Integration Network for Depression, St. Michael's Hospital, University Health Network, Toronto, Ont., Canada (Kennedy); and the Department of Psychiatry, University of Toronto, Toronto, Ont., Canada (Kennedy).

Competing interests: M. Leyton declares having received operating funds through his institution from GlaxoSmithKline in 2006-2007 to conduct a study on naltrexone. S. Kennedy reports grants from BMS, Janssen, Lundbeck, Pfizer, Servier, St. Jude Medical, the Ontario Brain Institute, Canadian Institutes of Health Research, Brain Canada, the Ontario Mental Health Foundation and the Ontario Research Fund; is on the advisory boards of and has received personal fees from Allergan, AstraZeneca, BMS, Janssen, Lundbeck, Pfizer, Servier, St. Jude Medical and Sunovion; has received speaker fees from Xian-Janssen; and has received research support from Brain Cells Inc and Clera. 


\section{References}

1. Kapur S, Phillips AG, Insel TR. Why has it taken so long for biological psychiatry to develop clinical tests and what to do about it? Mol Psychiatry 2012;17:1174-9.

2. Boksa P. A way forward for research on biomarkers for psychiatric disorders. J Psychiatry Neurosci 2013;38:75-7.

3. Finn ES, Shen $X$, Scheinost D, et al. Functional connectome fingerprinting: identifying individuals using patterns of brain connectivity. Nat Neurosci 2015;18:1664-71.

4. Kaufmann T, Alnaes D, Doan NT, et al. Delayed stabilization and individualization in connectome development are related to psychiatric disorders. Nat Neurosci 2017;20:513-515.

5. Gabard-Durnam LJ, Gee D, Goff B, et al. Stimulus-specific connectivity influences resting-state connectivity years later in human development: a prospective study. J Neurosci 2016;36:4771-84.

6. Lee T-H, Miernicki ME, Telzer EH. Families that fire together smile together: resting state connectome similarity and daily emotional synchrony in parent-child dyads. Neuroimage 2017;152:31-7.

7. Biazolo CE, Salum GA, Pan PM, et al. Functional connectome fingerprint: identifying individuals using patters of brain connectivity. Front Hum Neurosci 2017;11:47.

8. Downar J, Geraci J, Salomons TV, et al. Anhedonia and rewardcircuit connectivity distinguish nonresponders from responders to dorsomedial prefrontal repetitive transcranial magnetic stimulation in major depression. Biol Psychiatry 2014;76:176-85.

9. Manelis A, Almeida JRC, Stiffler R, et al. Anticipation-related brain connectivity in bipolar and unipolar depression: a graph theory approach. Brain 2016;139:2554-66.

10. Drysdale AT, Grosenick L, Downar J, et al. Resting-state connectivity biomarkers define neurophysiological subtypes of depression. Nat Med 2017;23:28-38.

11. Dunlop BW, Rajendra JK, Craighead WE, et al. Functional connectivity of the subcallosal cingulate cortex and differential outcomes to treatment with cognitive-behavioral therapy or antidepressant medication for major depressive disorder. Am J Psychiatry 2017;174:533-45.

12. Van Dam NT, O'Connor D, Marcelle ET, et al. Data-driven phenotypic categorization for neurobiological analyses: beyond DSM-5 labels. Biol Psychiatry 2017;81:484-94.

13. Lee T-H, Telzer EH. Negative functional coupling between right fronto-parietal and limbic resting state networks predicts increased self-control and later substance use onset in adolescence. Dev Cogn Neurosci 2016;20:35-42.

14. Luijten M, Schellekens AF, Kühn S, et al. Disruption of reward processing in addiction: an image-based meta-analysis of functional magnetic resonance imaging studies. JAMA Psychiatry 2017;74:387-98.

15. Nikolova YS, Knodt AR, Radtke SR, et al. Divergent responses of the amygdala and ventral striatum predict stress-related problem drinking in young adults: possible differential markers of affective and impulsive pathways of risk for alcohol use disorder. Mol Psychiatry 2016;21:348-56.

16. Leyton M. Altered dopamine transmission as a familial risk factor for addictions. Curr Opin Beh Sci 2017;13:130-8.

17. Cox SML, Jaworska N, Tippler M, et al. mGluR5 availability in emerging adults at risk for addictions: a high-resolution PET [11C]
ABP688 study [poster]. Canadian College of Neuropsychopharmacology meeting; 2017 June 7-9; Kingston.

18. Swartz JR, Williamson DE, Hariri AR. Developmental change in amygdala reactivity during adolescence: effects of family history of depression and stressful life events. Am J Psychiatry 2015;172:276-83.

19. Blackford JU, Allen AH, Cowan RL, et al. Amygdala and hippocampus fail to habituate to faces in individuals with an inhibited temperament. Soc Cogn Affect Neurosci 2013;8:143-50.

20. Sheffield JM, Kandala S, Tamminga CA, et al. Transdiagnostic associations between functional brain network integrity and cognition. JAMA Psychiatry 2017 10.1001/jamapsychiatry.2017.0669.

21. Sheffield JM, Kandala S, Burgess GC, et al. Cingulo-opercular network efficiency mediates the association between psychotic-like experiences and cognitive ability in the general population. Biol Psychiatry Cogn Neurosci Neuroimaging 2016;1:498-506.

22. Sheffield JM, Repovs G, Harms MP, et al. Fronto-parietal and cingulo-opercular network integrity and cognition in health and schizophrenia. Neuropsychologia 2015;73:82-93.

23. Palaniyappan L, Liddle PF. Does the salience network play a cardinal role in psychosis? An emerging hypothesis of insular dysfunction. J Psychiatry Neurosci 2012;37:17-27.

24. Weinberger DR, Radulescu E. Finding the elusive psychiatric "lesion" with 21st-century neuroanatomy: a note of caution. Am J Psychiatry 2016;173:27-33.

25. Chan SY, Loscazo J. The emerging paradigm of network medicine in the study of human disease. Circ Res 2012;111:359-74.

26. Iturria-Medina Y, Sotero RC, Toussaint PJ, et al. Early role of vascular dysregulation on late-onset Alzheimer's disease based on multifactorial data-driven analysis. Nat Commun 2016;7:11934.

27. Pascoal TA, Mathotaarachchi S, Shin M, et al. Synergistic interaction between amyloid and tau predicts the progression to dementia. Alzheimers Dement 2016 Dec. 23 [Epub ahead of print]. doi:10.1016/j.jaiz.2016.11.005.

28. Jack CR Jr, Wiste HJ, Weigand SD, et al. Age-specific and sexspecific prevalence of cerebral $\beta$-amyloidosis, taopathy, and neurodegeneration in cognitively unimpaired individuals aged 50-95 years: a cross-sectional study. Lancet Neurol 2017;16:435-44.

29. Lamberink HJ, Otte WM, Geerts AT, et al. Individualised prediction model of seizure recurrence and long-term outcomes after withdrawal of antiepileptic drugs in seizure-free patients: a systematic review and individual participant data meta-analysis. Lancet Neurol 2017 May 5 [Epub ahead of print]. doi:10.1016/ S1474-4422(17)30114-X.

30. Kennedy SH, Downar J, Evans KR. The Canadian Biomarker Integration Network in Depression (CAN-BIND): advances in response prediction. Curr Pharm Des 2012;18:5976-89.

31. Clementz BA, Sweeney JA, Hamm JP, et al. Identification of distinct psychosis biotypes using brain-based biomarkers. Am J Psychiatry 2016;173:373-84.

32. Insel T, Cuthbert B, Garvey M, et al. Research Domain Criteria (RDoC): toward a new classification framework for research on mental disorders. Am J Psychiatry 2010;167:748-51.

33. Chase HW, Fournier JC, Bertocci MA, et al. A pathway linking reward circuitry, impulsive sensation-seeking and risky decision-making in young adults: identifying neural markers for new interventions. Transl Psychiatry 2017;7:e1096. 